\title{
Dynamics observer for the longitudinal behavior of a wheelset on a roller rig
}

Christoph Schwarz ${ }^{1}$, Jonathan Brembeck ${ }^{1}$ and Benjamin Heckmann ${ }^{2}$ 


\section{Abstract}

The presented work illustrates a nonlinear model based observer synthesis for the longitudinal dynamics estimation of a wheelset. This approach offers some significant improvements, such as an enhanced adherence to prescribed brake distances and an advanced usability of the condition based maintenance of brake systems. The investigation of a single wheelset in a test rig environment enables for example the usage of an extended set of sensors to reliably validate the observers. The presented observer design process covers three main steps: identification of characteristic system disturbances, implementation of the nonlinear observer dynamics, and parametrization of the observer via multi-case optimization. Regarding the longitudinal dynamics the variations of the friction conditions in the wheel-rail interface and in the interface between brake pads and brake disc have a crucial influence. Therefore, a parameter estimator and a disturbance observer are implemented, since these methods allow for a specific consideration of these disturbances. The received results proof that the observers accurately estimate the system behavior and provide reliable information on the longitudinal dynamics.

\section{Keywords}

wheelset dynamics, railway modelling, observer design, laboratory testing

\footnotetext{
${ }^{1}$ Institute of System Dynamics and Control, German Aerospace Center (DLR), Germany

${ }^{2}$ Knorr-Bremse Rail Vehicle Systems, Germany

Corresponding author:

Christoph Schwarz, Institute of System Dynamics and Control, German Aerospace Center (DLR), Münchener Strasse 20, 82234 Wessling, Germany.

Email: christoph.schwarz@dlr.de
} 


\section{Introduction}

The trade-off between safety, comfort and wear is an essential aspect of many research activity that deals with railway technology. ${ }^{1,2}$ Regarding the longitudinal railway dynamics the wheel-rail interaction strongly influences all of the three criteria. Current traction and braking systems like wheel slide and skid protection already ease the trade-off to a certain extent. ${ }^{3}$ The results in Kurzeck et al. ${ }^{4}$ demonstrated a great potential of mechatronic control applications to reduce the wear of wheels and rails in terms of lateral dynamics. Hence, it is suggested that in the same way advanced brake control measures might reduce the wear and enhance the adherence to prescribed brake distances.

Most of these control algorithms require the knowledge of all system states. However, a direct measurement of all states is usually not feasible due to technical and economic reasons. Thus, the current longitudinal dynamics of the railway vehicle have to be determined by an observer which provides estimated information on each system state. Regarding the rough operating conditions of railway vehicles and the stringent safety requirements, the observer synthesis needs to be robust, reliable and accurate. To implement, test, and validate an observer in this novel field of application, it is reasonable to firstly restrict the complexity of the system. Therefore, the observer is designed for a single wheelset on a roller rig ${ }^{5}$, what additionally offers the advantage of an extended set of sensors to reliably validate the observer results. Finally, the observer design process covers three steps: identification of characteristic system disturbances, implementation of the nonlinear observer dynamics, and specification as well as parametrization of the observer.

The paper is organized as follows: The first section presents the configuration of a wheelset on the ATLAS (Advanced Test Laboratory for Adhesion based Systems) test rig of Knorr-Bremse in Munich, see Figure 1. Furthermore, the variations of the relevant parameters for the 


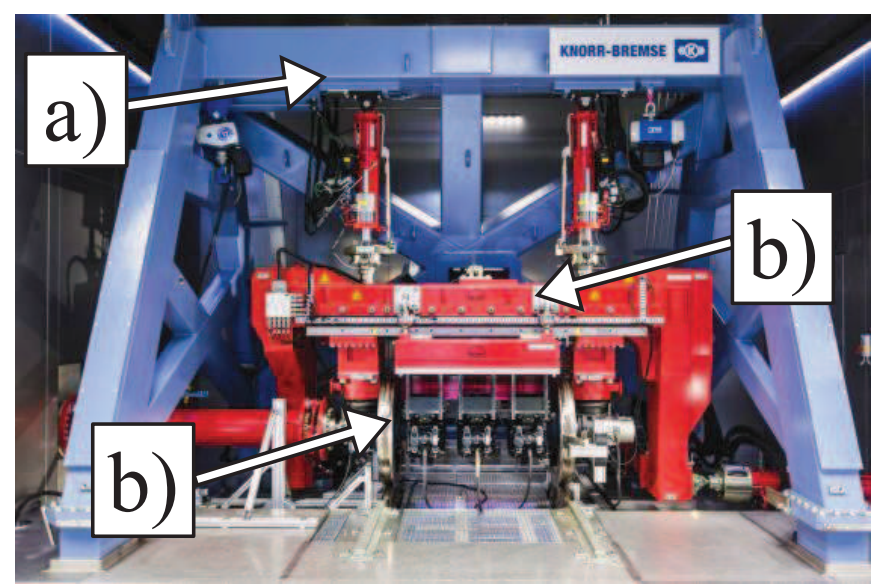

Figure 1. ATLAS test rig of Knorr-Bremse in Munich. ${ }^{6}$

longitudinal dynamics of a wheelset are identified, so that the observer design can systematically consider these parameters. Based on this system characterization three nonlinear simulation models are implemented, that are used as reference model and observer models, respectively. The following section describes two suitable observer methods, namely the parameter estimator and the disturbance observer. Afterwards, the two observers are optimized in a multi-case optimization ${ }^{7}$ with a set of measurement data recorded at the ATLAS test rig. In addition, promising results are illustrated that assure the accuracy, reliability, and robustness of the observers. The last section draws a conclusion and underlines the contributions to the field of railway technology research.

\section{Wheelset and test rig environment}

The synthesis of a model based observer begins with the identification of the observed system and its peculiarities. After that the observer models are implemented that cover exactly these characteristics in a suitable way. 


\section{Mechanical configuration of the sytem}

Regarding the wheelset on the roller rig in Figure 1 the system identification starts with a subdivision of the mechanical set-up into four main parts:

1. The outer structure serves as mounting and is considered to be fixed and rigid.

2. The inner structure represents to some extent a bogie frame. It is linked to the outer frame via two vertical cylinders, which can actively apply forces and in this way imitate different axle loads. Furthermore, it is connected with the roller rig housing via two horizontal, longitudinal cylinders on rail level. These cylinders are equipped with force sensors, so that they measure an equivalent to the longitudinal forces in the wheel-rail contact. The fifth cylinder is laterally mounted and can control a predefined hunting motion.

3. The wheelset is the centerpiece of the test rig and can be equipped with up to three axle brake units. Although Figure 1 depicts a driven wheelset, the presented work focuses on an undriven wheelset affected only by friction brakes.

4. The roller, which is hidden under the test rig floor, imitates the longitudinal motion of the wheelset, i.e. the roller angular velocity $\omega_{R}$ is proportional to the longitudinal velocity on a real track. By applying a suitable motor current on the roller, the inertia of an entire train can be imitated.

Another feature of the ATLAS test rig is the possibility to test the hard- and software components under realistic and alternating operating conditions such as rain, wind and different ambient temperatures. ${ }^{8}$ These functionalities are used later on to test the robustness and the reliability of the observers. 


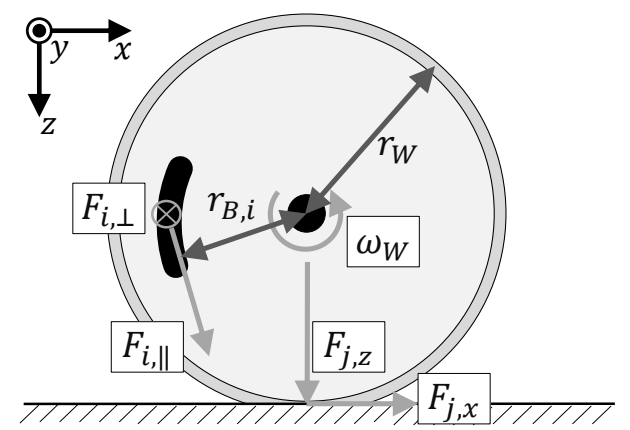

Figure 2. Relevant forces and positions of the wheelset on the roller rig.

Before the simulation models are implemented the variations of the most influencing parameters are identified that might significantly affect the dynamical behavior of the wheelset. Regarding the longitudinal motion, the relevant parameters are revealed by the moment equilibrium around the $y$-axis of the undriven wheelset, see Figure 2,

$$
J_{\mathrm{W}, y} \cdot \dot{\omega}_{W}=\sum_{i}\left|\boldsymbol{F}_{i, \|}\right| \cdot\left|\boldsymbol{r}_{\mathrm{B}, i}\right|+\sum_{j} F_{j, x} \cdot r_{\mathrm{W}}
$$

with $F_{i, \|}=\mu_{\mathrm{DC}, i} \cdot F_{i, \perp}$. The limitation of the longitudinal creep force is

$$
F_{j, x, \max }=\mu_{\mathrm{WR}, j} \cdot F_{j, z},
$$

with $i=1, \ldots, 3$ for the axle brake unit and $j=l, r$ representing the left and right wheel. In (1) $J_{\mathrm{W}, y}$, denotes the moment of inertia of the wheelset around the lateral axis and $\omega_{W}$ the angular wheel velocity. $\boldsymbol{F}_{i, \|}=\left[F_{i, \|, x} ; 0 ; F_{i, \|, z}\right]$ is the tangential friction force between brake disc and brake caliper and $F_{i, \perp}$ is the normal force. In addition, the forces in the wheel-rail interface are $\boldsymbol{F}_{j}=\left[F_{j, x} ; F_{j, y} ; F_{j, z}\right]$, with the longitudinal creep force $F_{j, x}$ and the normal force $F_{j, z}$. The other parameters of (1) and (2) are listed in Table 1 . The nominal values are mean values of different 


\begin{tabular}{|l|cc|rl|rl|}
\hline parameter & \multicolumn{2}{|c|}{ nominal } & \multicolumn{2}{|c|}{ abs. variation } & \multicolumn{2}{|c|}{ rel. variation } \\
\hline wheel radius $r_{\mathrm{W}}$ & 460 & $\mathrm{~mm}$ & -35 & $\mathrm{~mm}$ & -8 & $\%$ \\
\hline brake radius $\left|\boldsymbol{r}_{\mathrm{B}}\right|$ & 300 & $\mathrm{~mm}$ & \pm 15 & $\mathrm{~mm}$ & \pm 5 & $\%$ \\
\hline \multirow{2}{*}{ COF wheel-rail $\mu_{\mathrm{WR}}$} & \multirow{2}{*}{0.15} & & +0.25 & & +167 & $\%$ \\
& & & -0.1 & & -67 & $\%$ \\
\hline COF disc-caliper $\mu_{\mathrm{DC}}$ & 0.3 & & +0.15 & & +50 & $\%$ \\
-0.2 & & -67 & $\%$ \\
\hline wheel load $F_{j, z}$ & 80 & $\mathrm{kN}$ & \pm 8 & $\mathrm{kN}$ & \pm 10 & $\%$ \\
\hline
\end{tabular}

Table 1. Relevant parameters for the longitudinal dynamics with their maximum variations.

railway configurations and the variations are taken from the technical specification for interoperability of the European Union Agency for Railways. ${ }^{9}$ Regarding the wheel load the variation describes the difference between a wagon with and without passengers but neglects the dynamic wheel load deviation due to track irregularities. The two coefficients of friction (COF) between wheel and rail as well as between brake discs and brake calipers show the highest relative variations. Therefore, their impact on the longitudinal dynamics is taken into account in the assessment process of the observer method.

\section{Simulation models of the sytem}

There are three nonlinear models namely a multibody simulation model and two observer models. These models vary in the degree of complexity and, thus, are used for specific purposes. Nevertheless, all variants share some features. First of all, they are generated using the object-oriented and equation based modeling language Modelica. ${ }^{10}$ Modelica allows for a multi-physical design of the entire wheelset and test rig system. However, the influence of the pneumatic and electric components are neglected, since the focus of this work is on the mechanical interaction between the hardware components and their estimation. The second common aspect is that the friction interface between brake disc and brake caliper is 


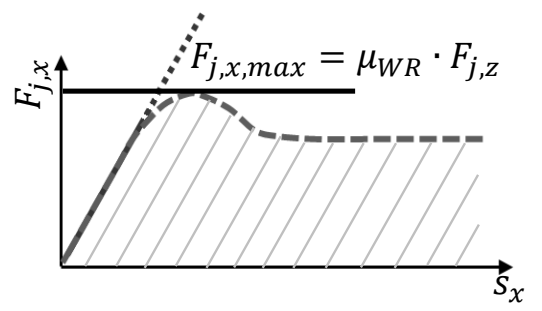

Figure 3. Longitudinal creep force $F_{j, x}$ over longitudinal slip $s_{x}$ according to Polach's contact formulation. ${ }^{11}$

modeled as a linear correlation, see (1). This simplification reduces the complexity and the computational effort. Another shared property is the nonlinear formulation of the wheel-rail contact according to Polach. ${ }^{11}$ This implementation provides a good trade-off between accuracy and computational efficiency and works well in acceleration and deceleration scenarios. The nonlinear relation between the longitudinal creep force $F_{j, x}$ and the longitudinal slip $s_{x}$, presented in Figure 3, results from the assumption, that $\mu_{\mathrm{WR}}$ decreases for an increasing slip velocity. As the focus is on the longitudinal dynamics, the lateral creep force as well as the creep torque are neglected.

Subsequent to the common aspects of the simulation models their differing properties are illustrated. The first model is a nonlinear multibody simulation model, see Figure 4. This system serves as a reference and can be used to simulate scenarios that might not be realized on the ATLAS test rig, like blocking wheels. The multibody model comprises all of the four mechanical parts listed above. Thus, it is composed of eight rigid bodies that have got 21 degrees of freedom (DOF), see Table 2. The names of the DOFs are related to the coordinate system shown in Figure 4.

In contrast to the reference system, the second and third model are used as a basis for the observer synthesis. To reduce the complexity and the 


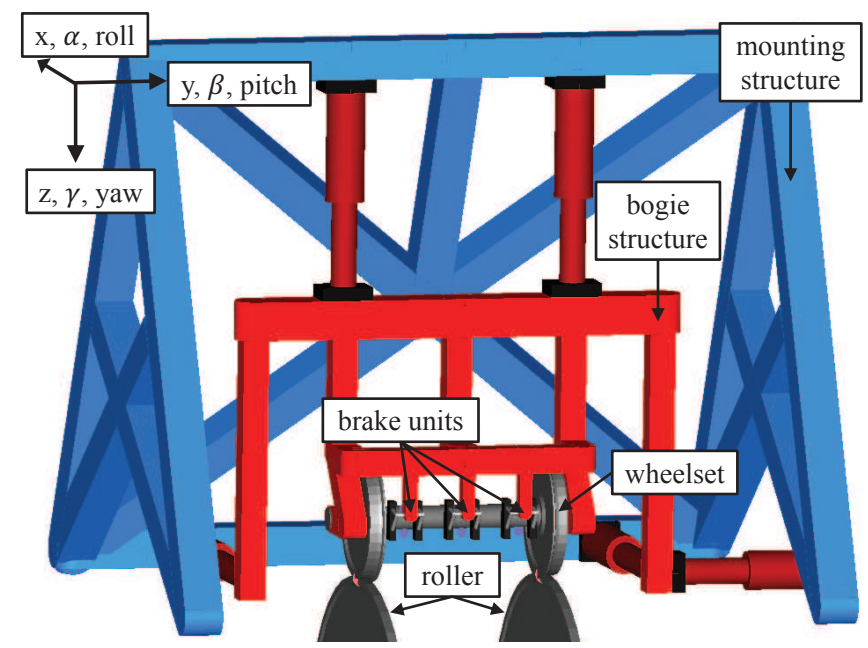

Figure 4. Detailed 3D multibody model of the wheelset on the test rig.

\begin{tabular}{|c|c|c|c|}
\hline & multibody model & full obs. model & min. obs. model \\
\hline bogie frame & $\begin{array}{c}x_{\mathrm{F}}, y_{\mathrm{F}}, z_{\mathrm{F}} \\
\alpha_{\mathrm{F}}, \beta_{\mathrm{F}}, \gamma_{\mathrm{F}}\end{array}$ & $z_{\mathrm{F}}$ & - \\
\hline wheelset guid. & $2 \cdot \beta_{\mathrm{G}, j}$ & - & - \\
\hline brake unit & $3 \cdot\left(z_{\mathrm{B}, i}, \beta_{\mathrm{B}, i}\right)$ & $\beta_{\mathrm{B}}$ & - \\
\hline wheelset & $\begin{array}{c}x_{\mathrm{W}}, y_{\mathrm{W}}, z_{\mathrm{W}} \\
\alpha_{\mathrm{W}}, \omega_{\mathrm{W}}, \gamma_{\mathrm{W}}\end{array}$ & $\omega_{\mathrm{W}}$ & $\omega_{\mathrm{W}}$ \\
\hline roller & $\omega_{\mathrm{R}}$ & $\omega_{\mathrm{R}}$ & $\omega_{\mathrm{R}}$ \\
\hline
\end{tabular}

Table 2. Overview on the bodies and degrees of freedom of the test rig models.

computational effort, both observer models describe the wheelset on the test rig as a $2 \mathrm{D}$ system in the $\mathrm{x}$-z-plane. Furthermore, $\beta_{\mathrm{F}}, x_{\mathrm{F}}, z_{\mathrm{B}}, x_{\mathrm{W}}$, and $z_{\mathrm{W}}$ are locked, since they turned out to be insignificant at least in the test rig environment. In the end, the system with four DOFs is named full observer model, see Table 2. The minimal observer model additionally neglects $z_{\mathrm{F}}$ and $\beta_{\mathrm{B}}$, so that only the two angular velocities $\omega_{\mathrm{W}}$ and $\omega_{\mathrm{R}}$ remain as DOFs. The two models are used for two different observer approaches, which are presented in the following section. 
The reference model is implemented using Modelica multibody components ${ }^{10}$, while the observer models are analytically formulated as nonlinear state space systems

$$
\dot{x}_{o}=f_{o}\left(x_{o}, u\right), \quad y=C_{o} \cdot x_{o},
$$

with the inputs $\boldsymbol{u} \in \mathbb{R}^{2 \times 1}$, the outputs $\boldsymbol{y} \in \mathbb{R}^{2 \times 1}$, and the system states $x_{o} \in \mathbb{R}^{n_{o} \times 1}$. The index $o=\mathrm{f}, \mathrm{m}$ denotes whether it is the full or the minimal observer model. The system inputs $\boldsymbol{u}$ are the brake pressure $p_{\mathrm{B}}$ of the axle brake units and the roller torque $\tau_{\mathrm{R}}$. The output vector $\boldsymbol{y}$ includes the angular velocity $\omega_{\mathrm{W}}$, which is usually measured in most railway vehicles. In addition, $\boldsymbol{y}$ includes $\omega_{\mathrm{R}}$, which represents the longitudinal speed on a real track. In modern trains at least a guess value of the longitudinal speed is usually provided by the wheel slip protection system. Regarding a running gear with driven wheelsets the presented method can directly be applied without structural adaptions, if the electrodynamic drive and brake currents of the motor are measurable. According to the different DOFs of the observer models the states of the full model are $\boldsymbol{x}_{\mathrm{f}}=\left[z_{\mathrm{F}} ; \dot{z}_{\mathrm{F}} ; \beta_{\mathrm{B}} ; \dot{\beta}_{\mathrm{B}} ; \omega_{\mathrm{W}} ; \omega_{\mathrm{R}}\right]$ and of the minimal model $\boldsymbol{x}_{\mathrm{m}}=\left[\omega_{\mathrm{W}} ; \omega_{\mathrm{R}}\right]$.

The dynamic vectors $\boldsymbol{f}_{o}\left(\boldsymbol{x}_{o}, \boldsymbol{u}\right)$ are generated using Lagrange's equations $^{12}$

$$
\frac{\mathrm{d}}{\mathrm{d} t}\left(\frac{\partial T_{o}}{\partial \dot{\boldsymbol{q}}_{o}}\right)^{T}-\left(\frac{\partial T_{o}}{\partial \boldsymbol{q}_{o}}\right)^{T}+\left(\frac{\partial V_{o}}{\partial \boldsymbol{q}_{o}}\right)^{T}=\boldsymbol{Q}_{o},
$$

with the kinetic energy $T_{o}$, the potential energy $V_{o}$, the generalized forces and torques $\boldsymbol{Q}_{o}$, and the generalized coordinates $\boldsymbol{q}_{o}$ with their time derivatives $\dot{\boldsymbol{q}}_{\mathrm{m}}=\left[\omega_{\mathrm{W}} ; \omega_{\mathrm{R}}\right]$ and $\dot{\boldsymbol{q}}_{\mathrm{f}}=\left[\dot{z}_{\mathrm{F}} ; \dot{\beta}_{\mathrm{B}} ; \omega_{\mathrm{W}} ; \omega_{\mathrm{R}}\right]$. The calculation of the terms in (4) is based on the procedure illustrated in Schwarz ${ }^{13}$. In the end, the specification of the system dynamics $\boldsymbol{f}_{o}\left(\boldsymbol{x}_{o}, \boldsymbol{u}\right)$ as well as the definition of the output vector $y$ are the basis for the observer synthesis. 


\section{Observer synthesis for a disturbed system}

After the detailed description of the test rig and wheelset environment the following subsections focus on the control theoretic methods. First of all, the observability and the observer principle are described. To specifically address the identified disturbances, the parameter estimator and the disturbance observer ${ }^{16}$ are presented.

\section{Theoretical background}

Before an observer can be reasonably implemented an observability check of the system (3) is necessary. In a control theoretic context observability means that the non-measured states can be reconstructed by the information provided by $y .{ }^{14}$ The observability analysis of a linearized model reveals that only the minimal model is observable for the selected output vector $\boldsymbol{y}=\left[\omega_{\mathrm{W}} ; \omega_{\mathrm{R}}\right]$. A more strict analysis using nonlinear observability criteria ${ }^{15}$ will be presented in a subsequent publication.

The basic principle of most observers is analog to a feedback control, where a desired system behavior shall be realized. The difference is that an observer does not affect the real system but the implemented model with the observer input $\boldsymbol{u}_{\text {obs }}$

$$
\boldsymbol{u}_{\mathrm{obs}}=\boldsymbol{L} \cdot \Delta \boldsymbol{y}, \quad \text { with } \quad \Delta \boldsymbol{y}=\boldsymbol{y}-\hat{\boldsymbol{y}}
$$

that depends on the one hand on the deviation between the real measurement signals $\boldsymbol{y}$ and the observed outputs $\hat{\boldsymbol{y}}$ and on the other hand on the matrix $L \in \mathbb{R}^{n_{\text {obs }} \times 2}$. If the feedback $\boldsymbol{u}_{\text {obs }}$ is properly defined, it minimizes $\Delta \boldsymbol{y}$ and in consequence ensures the convergence over time between the real, usually unknown states $\boldsymbol{x}$ and the observed states $\hat{x}$

$$
\dot{\hat{x}} \rightarrow \dot{x}, \quad \text { with } \quad \dot{\hat{x}}=f\left(\hat{x}, u, u_{\text {obs }}\right) .
$$




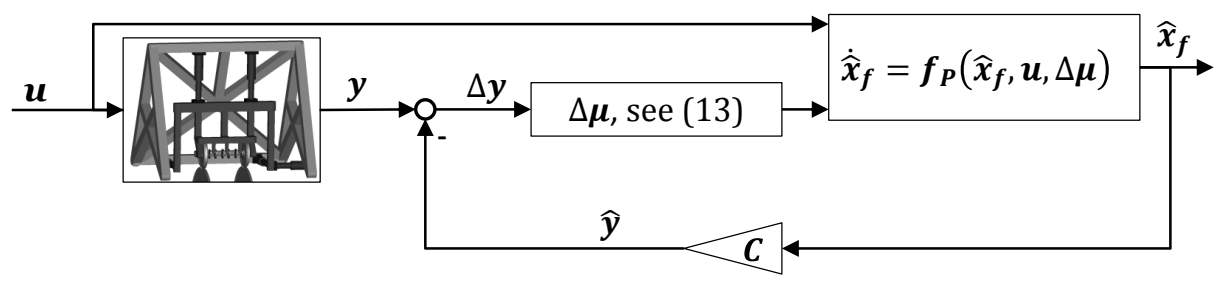

Figure 5. Scheme of the parameter estimator.

There are different ways to define the feedback correction, for example time discrete as well as time continuous Kalman filter methods, which consider the process noise and the measurement noise. ${ }^{17}$

\section{Parameter estimator}

In case of the parameter estimation, the full model is used as observer model, since the observability is not a necessary criterion for this approach. The feedback correction is

$$
\boldsymbol{u}_{\mathrm{obs}, \mathrm{P}}=\Delta \boldsymbol{\mu}=\left(\begin{array}{c}
\Delta \mu_{\mathrm{WR}} \\
\Delta \mu_{\mathrm{DC}}
\end{array}\right)=\boldsymbol{L}_{\mathrm{P}} \cdot\left[\Delta \boldsymbol{y}, \int \Delta \boldsymbol{y} \mathrm{d} t\right]^{T},
$$

with $L_{\mathrm{P}} \in \mathbb{R}^{2 \times 4}$ and the index $\mathrm{P}$ for parameter estimator. The integrated output deviation is considered to improve the convergence in stationary brake scenarios. Since $\boldsymbol{u}_{\mathrm{obs}, \mathrm{P}}$ directly affects the two COFs, the information on the friction conditions might not only be used for online control applications during a brake process but also for the long term condition based monitoring of brake components. However, only the qualitative information and not the exact values of the COFs should be consulted, since all disturbances in the system are traced back to deviations of $\mu_{\mathrm{WR}}$ and $\mu_{\mathrm{DC}}$. Another restriction is that the input $\Delta \mu_{\mathrm{DC}}$ only has got an effect on the system, if there is a brake demand $p_{\mathrm{B}}>$ 0. Furthermore, in scenarios with an almost ideal rolling motion, i.e. 


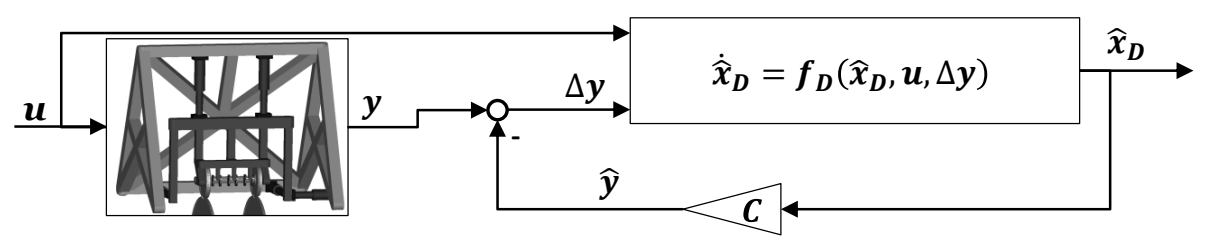

Figure 6. Scheme of the disturbance observer.

$s_{x}=0$, the input $\Delta \mu_{\mathrm{WR}}$ does not influence the dynamics either. As the most relevant longitudinal effects occur precisely during braking and accelerating phases with $s_{x} \neq 0$, the described restrictions do not significantly limit the performance of the parameter estimator.

\section{Disturbance observer}

The disturbance observer is the second method that is implemented and tested in the roller rig environment. ${ }^{16}$ In contrast to the parameter estimator the minimal model is used, since the observability is required in this case, where also the system states themselves are estimated. The disturbance observer introduces in a first step the variations $\Delta \mu_{\mathrm{WR}}$ and $\Delta \mu_{\mathrm{DC}}$ as disturbance states. The second step is to enlarge the state vector of the minimal observer model by the two disturbance states

$$
\hat{\boldsymbol{x}}_{\mathrm{D}}=\left(\begin{array}{c}
\hat{x}_{\mathrm{m}} \\
\Delta \mu_{\mathrm{WR}} \\
\Delta \mu_{\mathrm{DC}}
\end{array}\right) .
$$

The last step is to extend the system dynamics $f_{\mathrm{m}}$ by the disturbance dynamics $g_{\mathrm{D}}$ and add the feedback correction

$$
\dot{\boldsymbol{x}}_{\mathrm{D}}=\boldsymbol{f}_{\mathrm{D}}\left(\hat{\boldsymbol{x}}_{\mathrm{D}}, \boldsymbol{u}, \Delta \boldsymbol{y}\right)=\left(\begin{array}{c}
\boldsymbol{f}_{\mathrm{m}}\left(\hat{\boldsymbol{x}}_{\mathrm{D}}, \boldsymbol{u}\right) \\
\boldsymbol{g}_{\mathrm{D}}\left(\hat{\boldsymbol{x}}_{\mathrm{D}}, \boldsymbol{u}\right)
\end{array}\right)+\boldsymbol{L}_{\mathrm{D}}\left[\Delta \boldsymbol{y}, \int \Delta \boldsymbol{y} \mathrm{d} t\right]^{T},
$$


with $L_{\mathrm{D}} \in \mathbb{R}^{4 \times 4}$. Due to the influence of the feedback correction on the system states the drawbacks of the parameter estimator during pure roll phases vanish for the disturbance observer. Besides the parametrization of $L_{\mathrm{D}}$ the definition of $g_{\mathrm{D}}$ is an essential part of the observer design. The best results are received when the dynamic behavior of the disturbances can analytically be described. However, there is no generally accepted deterministic description of $\Delta \mu_{\mathrm{WR}}$ and $\Delta \mu_{\mathrm{DC}}$ due to unpredictable random influences like water or dirt in the friction interfaces. Therefore, an exponential function

$$
\boldsymbol{g}_{\mathrm{D}}=\left(\begin{array}{c}
T_{\mathrm{WR}} \cdot \Delta \mu_{\mathrm{WR}} \\
T_{\mathrm{DC}} \cdot \Delta \mu_{\mathrm{DC}}
\end{array}\right)
$$

is chosen representing a preferably generic characteristic. The exponential coefficients $T_{\mathrm{WR}}$ and $T_{\mathrm{DC}}$ as well as the parameters of $\boldsymbol{L}_{\mathrm{P}}$ and $\boldsymbol{L}_{\mathrm{D}}$ are optimized in the time domain, what is shown in the next section.

\section{Observer optimization and test results}

Using a numeric optimization for the parametrization is a reliable way to verify the observer concepts in the new application field of railway dynamics. The following investigations will systematically address the stability and robustness analysis of the observers in a control theoretic sense. Nevertheless, the robustness against different operating conditions as well as against changing system parameters is targeted in this section by executing a multi-case optimization ${ }^{7}$. The 14 investigated brake scenario cases vary in their initial velocities $\left(80,100,120\right.$ and $\left.160 \frac{\mathrm{km}}{\mathrm{h}}\right)$, their brake pressure $(1.5,2.7,3.6$ and 4.2 bar), the friction conditions of the wheelrail and disc-caliper contacts (dry, wet, only brake interface wet and only single brake interfaces wet) and the direction of the wheelset rotation.

There are different multi-case optimization types, which vary for example in their calculation of the objective function $z$. In this work $z$ 
is defined as the maximum value of all case objectives $z_{k}$

$$
\begin{aligned}
z & =\max _{k}\left(z_{k}\right), \quad \text { with } \\
z_{k} & =\frac{1}{t_{\text {end }, k}} \int_{0}^{t_{\text {end }, k}} c_{\mathrm{W}} \cdot\left|\Delta \omega_{\mathrm{W}}\right|+c_{\mathrm{R}} \cdot\left|\Delta \omega_{\mathrm{R}}\right|+c_{F} \cdot\left|\Delta F_{x}\right| \mathrm{d} t .
\end{aligned}
$$

The index $k=1, \ldots, 14$ represents the investigated case and $t_{\text {end, } k}$ is the respective test duration. The parameters $c_{\mathrm{W}}, c_{\mathrm{R}}$, and $c_{F}$ weight the absolute deviations between measured and observed variables, e.g. $\Delta \omega_{\mathrm{W}}=\omega_{\mathrm{W}}-\hat{\omega}_{\mathrm{W}}$. Using the maximum value of $z_{k}$ leads to an observer, that works well in all scenarios. The tuner variables of the optimization are in case of the parameter estimator the eight values of $L_{\mathrm{P}}$ and in case of the disturbance observer the 16 entries of $L_{\mathrm{D}}$ as well as $T_{\mathrm{WR}}$ and $T_{\mathrm{DC}}$.

After highlighting some structural aspects of the optimization problem, the tripartite framework of the simulation and optimization is illustrated in Figure 7. Firstly, the measurement data recorded at the ATLAS test rig is provided by look-up tables for each of the 14 optimization cases. Beside the system input data $\boldsymbol{u}$ and output data $\boldsymbol{y}$, which are available in a real application, the longitudinal creep force $F_{x}$ in the wheel rail contact is measured in the test rig environment. In the offline simulation environment the signals $u$ and $y$ are fed into the observer, which calculates the system information according to (6). This system simulation is executed for each of the 14 cases and the case objective $z_{k}$ is calculated and temporarily stored. Finally, to minimize $z$ the optimization algorithm adjusts the tuner parameters until the stop criterion of the optimization is fulfilled.

Exemplary observer results of an everyday stopping brake application with dry conditions are pictured in Figure 8. The upper plot shows the measurement data $\boldsymbol{u}$ and $\boldsymbol{y}$ that are used in the observers. The pressure $p_{\mathrm{B}}$ in the brake cylinders increases almost linearly in the beginning, remains constant in the middle part and drops as soon as the wheelset and the roller stop. The lower plot illustrates the force in the wheel-rail contact recorded 


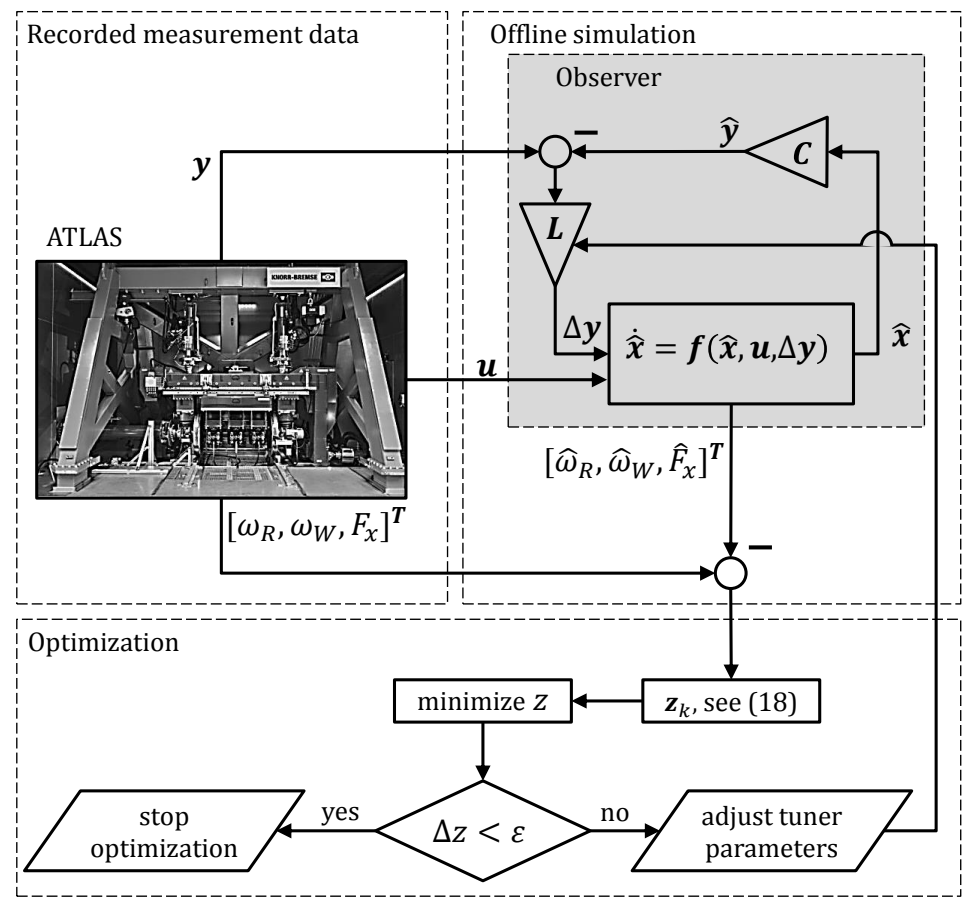

Figure 7. Framework of the multi-case optimization.

at the ATLAS test rig as well as the forces calculated by the observers. Both observer results perfectly imitate the increasing characteristic of the brake force over time that comes from an increasing friction coefficient between brake disc and brake pad. However, at the beginning of the brake process, when the brake pads and the brake disc firstly contact each other, there is a short-term oscillation at around $t=2 \mathrm{~s}$.

The results of a case with wet conditions shown in Figure 9 confirm the impressive accuracy of the observers. Again the oscillation at the beginning of the brake application occurs at about $t=1 \mathrm{~s}$, what might be eased by a rate limitation of the observer feedback. Even when the wheel-slide protection springs into action at $t=8 \mathrm{~s}$ the parameter estimator sufficiently performs. The disturbance observer does not completely reach 

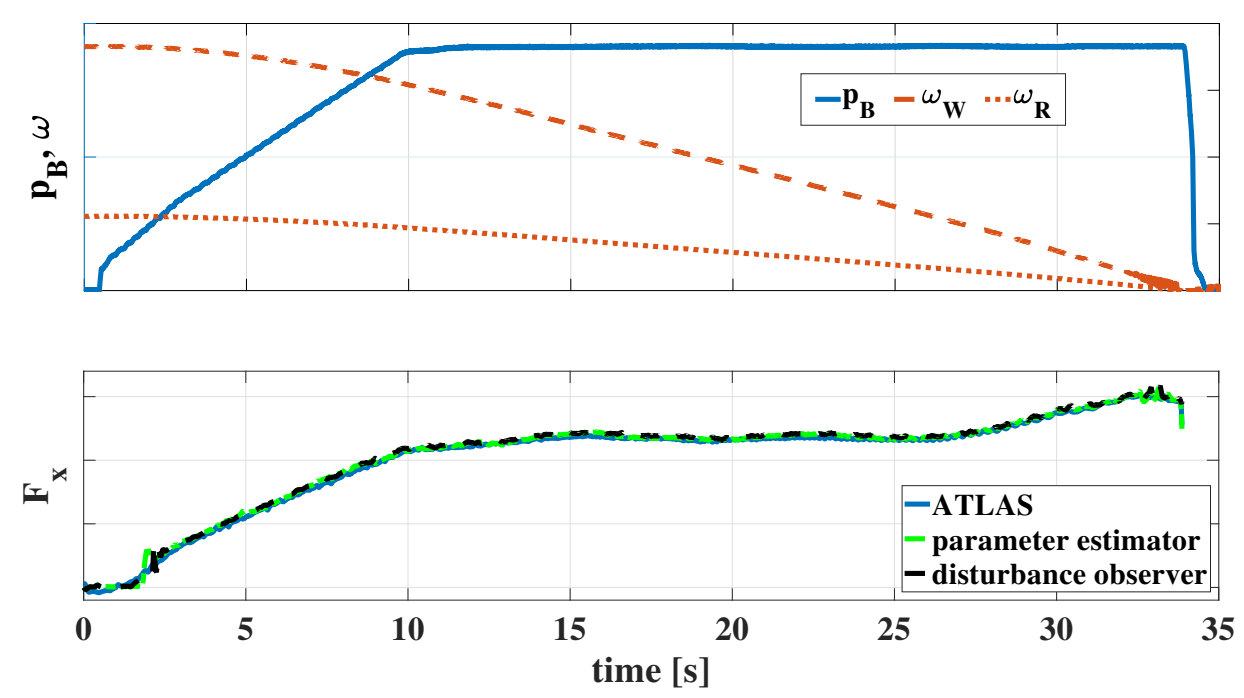

Figure 8. Observer input data (upper) and observer output data (lower) for a brake scenario with dry conditions.

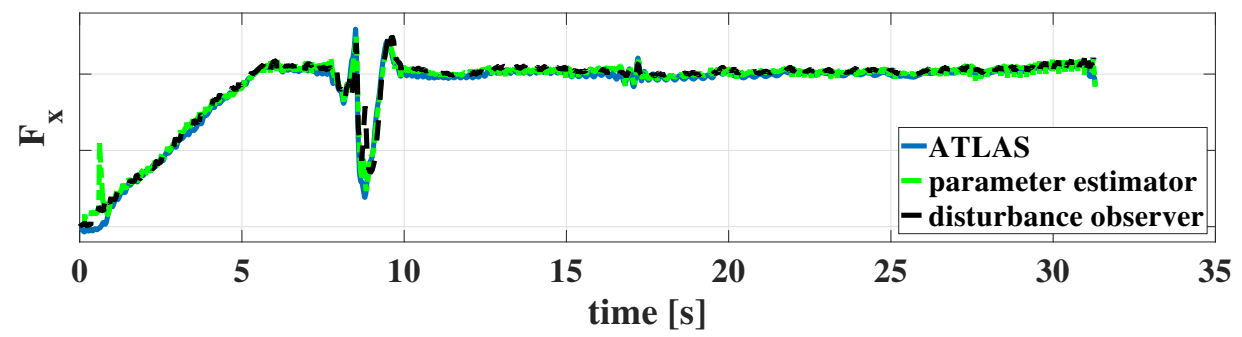

Figure 9. Observer results for the brake force $F_{x}$ in a brake scenario with wet conditions.

the minimum brake force but it catches the two positive peaks before and after the wheel-slide protection. All in all, the presented results certainly demonstrate that irrespective of the operating conditions both observers provide accurately estimated information, which can be used to enhance the longitudinal behavior. 


\section{Conclusions and contributions}

The previous sections give an overview on the observer synthesis for the longitudinal dynamics estimation of a wheelset. In detail, the setup of two observer models as well as the optimization of the observer parameters is described. The presented results show that the observers accurately estimate the system behavior and provide reliable information regarding the longitudinal dynamics like the longitudinal wheel-rail force. Exploiting this information in an advanced control setup facilitates a row of promising applications to improve safety, comfort and wear all at once. First of all, the uncertainty of brake distances caused by varying friction conditions might be minimized, what essentially enhances safety and comfort. In addition, condition based monitoring devices can be upgraded to improve the usability of condition based maintenance.

Nevertheless, there are some open tasks that might lead to an even higher accuracy of the observer results. One aspect is a physically motivated modeling of the disturbance dynamics $\boldsymbol{g}_{\mathrm{D}}$, for example dependent on the brake disc temperature or the wheelset rotational velocity. Another approach is the combination of state and parameter estimation that allows for a specific consideration of the quickly time-varying states and the slowly time-varying friction disturbances. ${ }^{18}$ Finally, the observers will be adapted to an entire train system and validated with data from track tests.

\section{Declaration of conflicting interests}

The author(s) declared no potential conflicts of interest with respect to the research, authorship, and/or publication of this article.

\section{Funding}

This work was supported by StMWi (StMWi grant number: MST-1308-0006// BAY 191/002), the Bavarian Ministry of Economic Affairs and Media, Energy and Technology, within the project DynORail. 


\section{References}

1. Bosso N, Gugliotta A and Zampieri N. Design and testing of an innovative monitoring system for railway vehicles. Proceedings of the Institution of Mechanical Engineers, Part F: Journal of Rail and Rapid Transit, 2016, 232 (2), 445-460.

2. Cruceanu C, Craciun C and Cruceanu I. Effects of Mechanical Wheel Slide Protection Devices Action on Railway Vehicles Braking Process. Applied Mechanics and Materials, 2015, 809810, 1085-1090.

3. Janicki J, Reinhard H and Rüffer M. Schienenfahrzeugtechnik. 3rd edn, 2013, Bahn Fachverlag.

4. Kurzeck B, Heckmann A, Kaiser I, Wesseler C and Keck A. Potenziale aktiv geregelter Losradfahrwerke im Hochgeschwindigkeitsverkehr - Ergebnisse aus dem DLR Projekt NextGeneration-Train. ZEVrail, 2014, Georg Siemens Verlag, 138 (8), 304-312.

5. Bruni S, Cheli F and Resta F. A model of an actively controlled roller rig for tests on full-size railway wheelsets. Proceedings of the Institution of Mechanical Engineers, Part F: Journal of Rail and Rapid Transit, 2001, vol. 215, 4: 277-288.

6. Knorr-Bremse AG. https://www.ausbildungsoffensive-bayern.de/mitgliedsunternehmen/knorrbremse-ag. online accessed July 162018.

7. Pfeiffer A. Optimization Library for Interactive Multi-Criteria Optimization Tasks. Proceedings of the 9th International Modelica Conference, 2012, Linköping University Electronic Press, 669-680.

8. Knorr-Bremse AG. Knorr-Bremse opens futuristic development center. press release, June 23, 2016.

9. Comission Decision of 21 February 2008 concerning a technical specification for interoperability relating to the rolling stock sub-system of the trans-European high-speed rail system. Official Journal of the European Union, 2008.

10. Heckmann A, Keck A, Kaiser I and Kurzeck B. The Foundation of the DLR RailwayDynamics Library: the Wheel-Rail-Contact. Proceedings of the 10th International Modelica Conference, 2014, Linköping University Electronic Press, 465-475.

11. Polach O. A fast wheel-rail forces calculation computer code. Vehicle System Dynamics, 2000, 33, 728-739.

12. Lanczos C The variational principles of mechanics. 4th edn, 1970, Dover Publications.

13. Schwarz C, Heckmann A and Keck A. Different Models of a Scaled Experimental Running Gear for the DLR RailwayDynamics Library. Proceedings of the 11th International Modelica Conference, 2015, Linköping University Electronic Press, 441-447. 
14. Lunze J. Regelungstechnik 2, Mehrgrössensysteme, Digitale Regelung. 6th edn, 2010, Springer-Verlag Berlin Heidelberg.

15. Adamy J Nichtlineare Systeme und Regelungen. 2nd edn, 2014, Springer-Verlag Berlin Heidelberg.

16. Föllinger O. Regelungstechnik, Einführung in die Methoden und ihre Anwendung. 10th edn, 2008, Hüthig-Verlag Heidelberg.

17. Brembeck J, Pfeiffer A, Fleps-Dezasse M, Otter M, Wernersson K and Elmqvist H. Nonlinear State Estimation with an Extended FMI 2.0 Co-Simulation Interface. Proceedings of the 10th International Modelica Conference, 2014, Linköping University Electronic Press, 53-62.

18. Plett GL. Sigma-point Kalman filtering for battery management systems of LiPB-based HEV battery packs Part 2: Simultaneous state and parameter estimation. Journal of Power Sources, 2006, 161, 1369-1384. 